\title{
All-Electron Study of Gradient Corrections to the Local Density Functional in Metallic Systems
}

\author{
Alexander Khein \\ Laboratory of Atomic and Solid State Physics, Cornell University, Ithaca, NY 14853 \\ D. J. Singh \\ Complex Systems Branch, Naval Research Laboratory, Washington, D.C. 20375-5000 \\ C. J. Umrigar \\ Theory Center and Laboratory of Atomic and Solid State Physics, Cornell University, Ithaca, NY 14853
}

(July 18, 1994)

\begin{abstract}
Using the all-electron Linearized Augmented Plane Wave (LAPW) method, we calculate the effect of including gradient corrections to the exchange correlation functional on the structural properties of the simple metal $\mathrm{Al}$, transition metals $\mathrm{Ta}, \mathrm{W}, \mathrm{Pt}$, and noble metals $\mathrm{Cu}, \mathrm{Ag}, \mathrm{Au}$. For all the systems studied, the local density approximation (LDA) yields bond-lengths that are too short and bulk moduli that are too large. The generalized gradient functional introduced by Perdew and Wang (PW91) yields corrections that are in the right direction (larger bond-lengths and smaller bulk moduli), but it frequently overcompensates, particularly for the heavier elements. The PW91 functional predicts the lattice constant and bulk modulus of $\mathrm{Al}$ and $\mathrm{Cu}$ more accurately than the LDA but yields values that are less accurate than the LDA for W, Pt and Au.
\end{abstract}

\section{INTRODUCTION}

The density functional method [1,2] provides a framework within which ground state properties of many physical systems can be calculated. While density functional theory is exact in principle, a practical implementation of the method requires the approximation of the electronic exchange correlation energy. The simplest and most widely used approximation to the exchange correlation energy is the local density approximation (LDA). For a given electronic density, the LDA replaces the true exchange correlation energy at each point in space by the exchange correlation energy of a homogeneous electron gas of the same density $[2,3]$.

In principle the LDA is only valid for an electron gas of slowly varying density. However, the LDA works surprisingly well in predicting ground state properties of atoms, molecules and solids with strongly inhomogeneous electron densities. The errors inherent in the LDA are similar among various systems. The limitations of a local formulation of exchange correlation are well known and here we mention only a few of them [4]. The LDA overestimates the binding energies of molecules and solids while underestimating the magnitudes of total energies. In atomic systems the LDA tends to overestimate the first ionization energy and underestimate $s p$ and $s d$ transfer energies. The LDA predicts a non magnetic FCC phase of bulk iron instead of the correct ferromagnetic BCC phase [5-7]. In bulk solids the LDA tends to overestimate bulk moduli and underestimate equilibrium lattice constants [4,8].

Recent attempts to improve the LDA have focused on the generalized gradient approximation (GGA) in which the exchange correlation functional incorporates terms containing density gradients [9-12]. Merits of a GGA approach include the ease with which a functional can be implemented in an existing code and the low computational cost of computing density gradients. In this article we focus on the GGA recently proposed by Perdew and Wang (PW91) $[8,12,13]$. The functional is based on a real space cut-off of the exchange correlation hole about an electron. The cut off ensures that the functional obeys known sum rules of the exchange correlation hole. This functional is also constructed to satisfy many of the scaling relations [14] for the exchange correlation energy [12,13].

Recent calculations incorporating the GGA have suggested that although no existing functional yields a uniform improvement over the LDA, recently proposed GGA functionals are promising. Total and ionization energies of atoms, binding energies and vibration frequencies of molecules are found to improve over the LDA [8,15,16]. Lattice constants, bulk moduli and cohesive energies of simple metals and selected transition metals, particularly the $3 \mathrm{~d}$ series, are found to improve when the GGA is used [8,17-20]. In particular, the GGA can fix the large discrepancy in lattice constant prevalent in the LDA calculations of the alkali metals [8]. In accordance with experiment, the GGA predicts the correct BCC ground state of iron [20-23]. Ortiz and Ballone investigated the bonding properties of group IIa, IIb 
homo-nuclear dimers using the GGA and found the GGA to yield better bonding energies, equilibrium distances and vibration frequencies than the LDA [24].

Recent work on semiconductors indicates that while cohesive energies are improved over the LDA, lattice constants and bulk moduli are significantly worse when the GGA is used [25,26]. There have been mixed reports of whether the GGA improves the ground state properties of the $4 d$ and $5 d$ transition metals [18,27-31]. Since the GGA functionals are particularly sensitive to charge inhomogeneities, it is has been noted that calculations free of shape approximations in the potential and charge density are important to accurately describe the effect of the GGA [8].

The methods employed to perform calculations can have a strong influence on the final results, particularly when a GGA functional is used. Consequently there is a spread in the results quoted by various authors, presumably due to the approximations used in their methods. Typically calculations are performed that incorporate certain approximations such as the pseudopotential approximation, shape approximations to the potential or charge density, or not fully taking account of relativistic effects. We use a well converged, all-electron technique free of shape approximations to the potential and charge density. The core electrons are fully relativistic while the valence electrons are semi-relativistic. When necessary, we employ local orbitals [32] to guarantee proper treatment of extended core states. Since our results are free of common approximations they are expected to more reliably reflect the limitations of the functionals being tested. In this paper we compare the results of our calculations of equilibrium lattice constants and bulk moduli for the metals, Al, Cu, Ag, Ta, W, Pt, and Au, using the LDA and the PW91 functionals. For comparison we include the results from recent work incorporating the PW91 functional.

\section{METHOD}

Our calculations are performed using the all electron linearized augmented plane wave (LAPW) method with fully relativistic cores. The details of the LAPW method are given elsewhere [33,34]; here we focus on aspects relevant to the present calculations. Our calculations for total energy are well converged with respect to both $\mathbf{k}$ point number and basis size. We used 60, $70 \mathrm{k}$ points [35] in the irreducible Brillouin zone for the FCC and BCC structures respectively, and a plane wave cut-off of $K_{\mathrm{max}} R_{\mathrm{mt}}=9$ where $K_{\mathrm{max}}$ is the reciprocal lattice vector cut-off and $R_{\mathrm{mt}}$ is the radius of the muffin tin sphere. For Ta, changing $K_{\max } R_{\mathrm{mt}}$ from 9 to 10 or increasing the $\mathbf{k}$ point number from 70 to 112 gave an energy change of only $0.5 \mathrm{mRy}$. It is important to note that the muffin tin is used to define the augmentation functions for the plane waves and in no way implies a shape restriction to the potential or charge density which are allowed to be completely general. The muffin tin sphere radius is chosen so that at the smallest lattice constant considered, the muffin tin spheres nearly touch. Tests were performed that indicated our results are insensitive to the choice of $R_{\mathrm{mt}}$. For instance, near the experimental lattice constant, changing the muffin tin radius of Au from 2.5 to 2.1 Bohr resulted in only a $2 \mathrm{mRy}$ change in total energy. At the muffin tin sphere boundary plane waves are matched continuously through the first derivative to radial solutions of Schrodinger equation. Consequently the PW91 exchange correlation potential is discontinuous at the muffin tin sphere boundary since it contains second derivatives of the charge density. LAPW calculations for iron have shown that small discontinuities do not affect the quality of the calculation [22]. We monitored the discontinuity in the potential for the GGA calculation and found that it was never larger than 0.15Ry. Typical values of the discontinuity were in the range of $0.03-0.07$ Ry. Energy parameters for the LAPW basis functions were set near the center of their respective bands using the output at the theoretical equilibrium lattice constant as a reference. Tests were performed to monitor the sensitivity of the calculation to the choice of energy parameters. We find that reasonable choices for the parameters yield identical results irrespective of the particular parameter values. For calculations of $\mathrm{Cu}, \mathrm{Ag}$, $\mathrm{Ta}, \mathrm{W}, \mathrm{Pt}$ and $\mathrm{Au}$ we used the local orbital extension of the LAPW method [32]. Calculations of Ta and W were made with the $5 p$ states in the valence. The local orbitals provided variational freedom to take these states into account allowing the use of another energy parameter for any residual $p$ character in the other valence states. The $3 p$ states of $\mathrm{Cu}, 4 p$ states of $\mathrm{Ag}$ and $5 p$ states of $\mathrm{Pt}$ and $\mathrm{Au}$ were calculated in the core region. For these elements, the local orbitals allow the basis set to have adequate variational freedom to orthogonalize valence states to the extended $p$ states in the core. The extended $p$ states of these metals are calculated in both core and valence, the latter serving to orthogonalize the valence states to the core $p$ states while the former is used in the calculation of the total energy. Without a local orbital extension the valence states are not strictly orthogonal to the core states and the latter can have adverse effects on the quality of the calculation. The LDA functional we use is the recent parameterization of Perdew and Wang [36]. 


\section{RESULTS AND DISCUSSION}

The results of our calculations are summarized in Table [1]. The error in lattice constant and bulk modulus relative to experiment is given in Figures [1] and [2] where we list the metals we studied in order of increasing atomic weight. For the purpose of comparison we include recent results for $\mathrm{Na}$ that were performed by one of the authors (DJS) using the LAPW method [8]. It is evident that in all cases, the LDA underestimates the lattice constant, the error being particularly large for $\mathrm{Na}$, and overestimates the bulk modulus. Except for $\mathrm{Na}$, the GGA overcorrects the lattice constant predicted by the LDA. The PW91 GGA fixes the LDA errors in Na. The bulk modulus is brought to within $4 \%$ of experiment and the lattice constant error is reduced to $0.1 \%$. A similar reduction of LDA errors occurs for $\mathrm{Al}$, particularly for the bulk modulus error which drops from $14 \%$ to $1.4 \%$. There have been several reports that the GGA can reduce errors in ground state properties of the $3 d$ metals [20,28,30]. We find improvement over the LDA for FCC Cu with the error in bulk modulus reduced from $43 \%$ to $13 \%$ while the error in lattice constant is reduced from $2.6 \%$ to $0.15 \%$.

While the error in the bulk modulus of the $4 d$ metal $\mathrm{Ag}$ is reduced by nearly a factor of two, the PW91 functional overcompensates the LDA lattice constant error without any improvement. For Ta, the lightest $5 d$ metal considered, the GGA improves the equilibrium properties. For the heaviest $5 d$ series metals, W, Pt and Au, we find PW91 gives overcompensated results that are in worse agreement with experiment than the LDA values. The error in the GGA result for $\mathrm{W}, \mathrm{Pt}$ and $\mathrm{Au}$ grows with increasing atomic number.

Finally, we compare our results with recent work incorporating the PW91 GGA. Our results for Al and the results of Ref. [8] for Na differ from those of Ref. [19]. The data from Ref. [19] seems to amplify the errors inherent in both the LDA and the GGA by predicting very small lattice parameters and overly large bulk moduli in the LDA with large overcompensation from PW91. Presumably the difference in the results arises from the choice of method. While we perform a full-potential all-electron calculation, Ref. [19] uses a pseudopotential approach. In Ref. [28] the full potential LMTO method was used to calculate the ground state properties of the $3 d, 4 d$ and $5 d$ transition metals. The results of Ref. [28] for Pt are in good agreement with ours but their results for Ta and W are not. The LMTO method used by Ref. [28] does not include a means by which the extended semi-core $5 p$ states can orthogonalize to the valence states. This is important for transition metals on the left side of the periodic table but less important for elements such as Pt that are further to the right. This is because the semi-core states contract into the core with increasing atomic number. The core and valence electrons are treated semi-relativistically in Ref. [28] while we treat the core electrons fully relativistic with semi-relativistic valence. We have tested the effect of the spin-orbit interaction in the core of $\mathrm{W}$ by calculating the lattice constant and bulk modulus within the LDA using a semi-relativistic core. We find no change from the fully relativistic calculation thus eliminating this as a candidate explanation for the difference.

We conclude that the PW91 GGA offers an improvement over the LDA for the light metal Al, the $3 d$ metal Cu and the light $5 d$ transition metal Ta. The PW91 GGA overcompensates for the errors in the metals Ag, W, Pt and $\mathrm{Au}$ leading to results that do not improve upon the LDA or are in worse agreement with experiment than the LDA.

\begin{tabular}{|c|c|c|c|c|c|c|}
\hline \multirow[t]{2}{*}{ Crystal } & \multirow[t]{2}{*}{ Property } & \multicolumn{2}{|c|}{ Present work } & \multicolumn{2}{|c|}{ Previous Calculations } & \multirow[t]{2}{*}{ experiment ${ }^{\epsilon}$} \\
\hline & & LDA & PW91 & LDA & PW91 & \\
\hline $\mathrm{Al}$ & $\begin{array}{c}a_{0}(\text { a.u. }) \\
B_{0}(\text { Mbar })\end{array}$ & $\begin{array}{c}7.52(-1.7) \\
83.9(14)\end{array}$ & $\begin{array}{c}7.74(1.2) \\
72.6(-1.4)\end{array}$ & $\begin{array}{c}7.43^{a} \\
87.65^{a}\end{array}$ & $\begin{array}{c}8.03^{a} \\
61.07^{a}\end{array}$ & $\begin{array}{l}7.65 \\
73.6\end{array}$ \\
\hline $\mathrm{Cu}$ & $\begin{array}{c}a_{0}(\text { a.u. }) \\
B_{0}(\mathrm{Mbar})\end{array}$ & $\begin{array}{c}6.65(-2.6) \\
192(43) \\
\end{array}$ & $\begin{array}{c}6.84(0.15) \\
151(13) \\
\end{array}$ & $\begin{array}{l}6.73^{b} \\
183^{b} \\
\end{array}$ & & $\begin{array}{l}6.83 \\
134 \\
\end{array}$ \\
\hline $\mathrm{Ag}$ & $\begin{array}{c}a_{0}(\text { a.u. }) \\
B_{0}(\text { Mbar })\end{array}$ & $\begin{array}{c}7.56(-1.9) \\
139(35)\end{array}$ & $\begin{array}{l}7.88(2.2) \\
84.8(-18)\end{array}$ & $\begin{array}{l}7.61^{c} \\
137^{c}\end{array}$ & & $\begin{array}{l}7.71 \\
103\end{array}$ \\
\hline $\mathrm{Ta}$ & $\begin{array}{c}a_{0}(\text { a.u. }) \\
B_{0}(\text { Mbar })\end{array}$ & $\begin{array}{c}6.16(-1.1) \\
224(9.8)\end{array}$ & $\begin{array}{c}6.28(0.80) \\
197(-3.4)\end{array}$ & $\begin{array}{l}6.07^{d} \\
187^{d} \\
\end{array}$ & $\begin{array}{l}6.21^{d} \\
175^{d} \\
\end{array}$ & $\begin{array}{l}6.23 \\
204 \\
\end{array}$ \\
\hline W & $\begin{array}{c}a_{0}(\text { a.u. }) \\
B_{0}(\text { Mbar })\end{array}$ & $\begin{array}{c}5.94(-0.50) \\
337(2.1) \\
\end{array}$ & $\begin{array}{c}6.02(0.84) \\
307(-7.0) \\
\end{array}$ & $\begin{array}{l}5.87^{d} \\
305^{d}\end{array}$ & $\begin{array}{l}5.98^{d} \\
271^{d} \\
\end{array}$ & $\begin{array}{l}5.97 \\
330 \\
\end{array}$ \\
\hline $\mathrm{Pt}$ & $\begin{array}{c}a_{0} \text { (a.u.) } \\
B_{0}(\text { Mbar })\end{array}$ & $\begin{array}{c}7.37(-0.54) \\
307(8.1) \\
\end{array}$ & $\begin{array}{l}7.51(1.3) \\
246(-13) \\
\end{array}$ & $\begin{array}{l}7.36^{d} \\
306^{d}\end{array}$ & $\begin{array}{l}7.51^{d} \\
263^{d}\end{array}$ & $\begin{array}{l}7.41 \\
284 \\
\end{array}$ \\
\hline $\mathrm{Au}$ & $\begin{array}{c}a_{0}(\text { a.u. }) \\
B_{0}(\text { Mbar })\end{array}$ & $\begin{array}{c}7.66(-0.65) \\
198(12)\end{array}$ & $\begin{array}{l}7.86(1.9) \\
142(-20)\end{array}$ & $\begin{array}{l}7.69^{c} \\
189^{c}\end{array}$ & & $\begin{array}{l}7.71 \\
177\end{array}$ \\
\hline
\end{tabular}


TABLE I. LAPW calculations of the lattice constant and bulk modulus using the Perdew-Wang exchange-correlation functional (PW91) [12] and LDA. Numbers in parentheses indicate percentage error relative to experiment.
a. Ref. [19]
b. Ref. [37]
c. Ref. [38]
d. Ref. [28]
e. Ref. [39] 
FIG. 1. Relative error in lattice constants. Results from other work are from Ref. [19] for Na and Al and from Ref. [28] for $\mathrm{Ta}, \mathrm{W}$ and Pt. Results for $\mathrm{Na}$ with filled circles and triangles are from Ref. [8]. Note that there is nearly a complete overlap of data for Pt between the present calculation and that of Ref. [28].

FIG. 2. Relative error in bulk moduli. Results from other work are from Ref. [19] for $\mathrm{Na}$ and $\mathrm{Al}$ and from Ref. [28] for Ta, $\mathrm{W}$ and Pt. Results for $\mathrm{Na}$ with filled circles and triangles are from Ref. [8]. Note that there is nearly a complete overlap of data between the present calculation and that of Ref. [28] for LDA Pt.

\section{ACKNOWLEDGMENTS}

Work at Cornell and at the Naval Research Laboratory, was supported by the Office of Naval Research. The calculations were performed on the IBM SP1 computer at the Cornell Theory Center.

[1] P. Hohenberg and W. Kohn, Phys. Rev. 136, B864 (1964)

[2] W. Kohn and L. J. Sham, Phys. Rev. 140, A1133 (1976)

[3] S. Lundqvist and N. H. March ed., Theory of the Inhomogeneous Electron Gas, (Plenum Press, New York, 1983)

[4] R. O. Jones and O. Gunnarsson, Rev. Mod. Phys. 61, 689 (1989)

[5] C. S. Wang, B. M. Klein and H. Krakauer, Phys. Rev. Lett. 54, 1852 (1985)

[6] K. B. Hathaway, H. J. F. Jansen and A. J. Freeman, Phys. Rev. B 31, 7603 (1985); H. J. F. Jansen, K. B. Hathaway and A. J. Freeman, ibid. 30, 6177 (1984)

[7] H. J. F. Jansen and S. S. Peng, Phys. Rev. B 37, 2689 (1988)

[8] J. P. Perdew, J. A. Chevary, S. H. Vosko, K. A. Jackson, M. R. Pederson, D. J. Singh and C. Fiolhhais, Phys. Rev. B 46, $6671(1992)$

[9] D. C. Langreth and M. J. Mehl, Phys. Rev. B 28, 1809 (1983)

[10] J. P. Perdew, Phys. Rev. B 33, 8822 (1986); erratum ibid. 34, 7406 (1986)

[11] J. P. Perdew and Y. Wang, Phys. Rev. B 33, 8800 (1986)

[12] J. P. Perdew in Electronic structure of solids '91, edited by P. Ziesche and H. Eschrig (Akademie Verlag, Berlin, 1991)

[13] J. P. Perdew, Physica B 172, 1 (1991)

[14] See C.J. Umrigar and X. Gonze, published in High Performance Computing and its Application to the Physical Sciences, proceedings of the Mardi Gras '93 Conference, edited by D. A. Browne et al, (World Scientific, Singapore, 1993) and references therein.

[15] A. D. Becke, J. Chem. Phys. 96, 2155 (1992); ibid. 97, 9173 (1992)

[16] B. G. Johnson, P. M. W. Gill and J. A. Pople, J. Chem. Phys. 98, 5612 (1993)

[17] D. J. Singh and J. Ashkenazi, Phys. Rev. B 46, 11570 (1992)

[18] N. A. W. Holzwarth and Y. Zeng, Phys. Rev. B 49, 2351 (1994)

[19] Y. Juan and E. Kaxiras, Phys. Rev. B 48, 14944 (1993)

[20] T. C. Leung, C. T. Chan and B. N. Harmon, Phys. Rev. B 44, 2923 (1991)

[21] J. Zhu, X. W. Wang and S. G. Louie, Phys. Rev. B 45, 8887 (1992)

[22] D. J. Singh, W. E. Pickett and H. Krakauer, Phys. Rev. B 43, 11628 (1991)

[23] T. Asada and K. Terakura, Phys. Rev. B 46, 13599 (1992)

[24] G. Ortiz and P. Ballone, Phys. Rev. B 43, 6376 (1991)

[25] G. Ortiz, Phys. Rev. B 45, 11328 (1992)

[26] Claudia Fillipi, Submitted to Phys. Rev. B., (1994)

[27] M. Körling and J. Häglung, Phys. Rev. B 45, 13293 (1992)

[28] V. Ozoliņš and M. Körling, Phys. Rev B 48, 18304 (1993)

[29] B. Barbiellini, E. G. Moroni and T. Jarlborg, J. Phys. Condens. Matter 2, 7597 (1990)

[30] P. Bagno, O. Jepsen and O. Gunnarsson, Phys. Rev. B 40, 1997 (1989)

[31] A. Garcia, C. Elsässer, J. Zhu, S. G. Louie and M. L. Cohen, Phys. Rev. B 46, 9829 (1992)

[32] D. Singh, Phys. Rev. B 43, 6388 (1991)

[33] O. K. Andersen, Phys. Rev. B 12, 3060 (1975)

[34] D. J. Singh, Planewaves, Pseudopotential, and the LAPW Method (Kluwer Academic Publishers, Boston 1994), and references therein

[35] H.J. Monkhorst and J. D. Pack, Phys. Rev. B 13, 5188 (1976); ibid. 16, 1748 (1977) 
[36] J. P. Perdew and Y. Wang, Phys. Rev. B 45, 13244 (1992)

[37] Z. W. Lu, S. -H. Wei and Alex Zunger, Phys. Rev. B 41, 2699 (1990)

[38] K. Terakura, T. Oguchi, T. Mohri and K. Watanabe, Phys. Rev. B 35, 2169 (1987)

[39] A. J. Moses, The Practical Scientists Handbook (Van Nostrand Reinhold Company, New York 1978) 